\title{
The Adaptation Approach for Affective Game-Based Assessment
}

\author{
Sintija Petroviča ${ }^{1}$, Alla Anohina-Naumeca ${ }^{2}$ \\ ${ }^{1,2}$ Riga Technical University, Latvia
}

\begin{abstract}
Game-based learning as a learning approach has been popular for ages; however, game-based assessment as a trend started to evolve only few years ago. Since knowledge assessment is more associated with negative emotions, systems intended to assess knowledge should take into consideration emotions as well. The analysis of existing studies shows that systems with integrated game-based assessment seldom utilise learner's emotions for provision of adaptation. The main aim of the present paper is to introduce an adaptation approach for to affective game-based assessment.
\end{abstract}

Keywords - Adaptation, emotions, game-based assessment.

\section{INTRODUCTION}

The popularity of digital games has drawn researchers' attention to the development of game-based educational settings to enhance learner's knowledge, skills, and other characteristics. The reasons behind this interest are different, e.g., games increase enjoyment, involvement, and motivation, as well as they influence emotions. Playing games is an emotional process and, at the same time, emotions are an important factor for successful learning. However, learners usually differ regarding their interests, preferences, and personality. All of the mentioned aspects could be analysed to provide adaptation in a learning environment [1].

Already in 2009, Conati and Maclaren argued that one of the reasons why research in the field of educational games does not provide hard evidence regarding the effectiveness of games as educational tools is because most of the existing games do not respond to specific needs of individual learners [2]. However, the analysis of recent studies still proves that learners' differences are seldom taken into account in educational games, and emotions as an adaptation source are used even rarer. Therefore, an improvement of adaptability in games could lead to higher learning results [3].

Games designed for the knowledge assessment as a direction have emerged only recently. The first educational game for the knowledge assessment SimCityEDU: Pollution Challenge! was developed in 2014 by GlassLab [4]. Currently, educational games with an aim of assessing knowledge are gaining increasing popularity. Traditionally, learner's knowledge is evaluated after the gameplay and this can be a time-consuming process; therefore, additional tools of the knowledge assessment should be developed and offered to learners as well [5]. Therefore, developers of educational games have realised that games can be used for assessment purposes. In general, knowledge assessment is more associated with negative emotions. High anxiety before an assessment (tests and/or exams) is a quite common phenomenon [6]. Thus, systems intended for the knowledge assessment should analyse affective data as well. Although many people get relaxed and can concentrate on the assessment, unfortunately, there are still people who cannot regulate their negative emotions and due to this reason they fail in exams [7]. Therefore, educational environments (e.g., games) designed to assess learners' knowledge should also take into account their emotions.

The analysis of existing games designed for knowledge assessment reveals that learner's (or player's) emotions are seldom used with the aim of providing adaptation. In the best case, developers give recommendations how to use identified emotions for adaptation purposes [8]. The main aim of this paper is to present an adaptation approach, which takes into consideration learner's differences for the (affective) assessment

The structure of the paper is as follows. Section II introduces such concepts as game-based learning and gamebased assessment. Section III provides the analysis of existing research related to adaptation to emotions in game-based environments. The next section describes the proposed adaptation approach for the knowledge assessment. Section V outlines the implementation aspects of the developed adaptation approach as part of the game. Conclusions are given at the end of the paper.

\section{GAME-BASED LEARNING AND ASSESSMENT}

Usage of games for educational purposes is called gamebased learning [1]. Nowadays, it is a subject of increasing attention as games include goals, rules, learning content, achievements, outcomes, and feedback (the main elements of learning process). Additionally, games develop problem solving skills, present challenges and competitions, as well as promote interaction between players. They provide basic preconditions for learning by supporting engagement, motivation, creativity, social interaction, and emotions [9], [10]. Since engagement and motivation are critical aspects for improving learning outcomes then facilitation of positive emotions has become one of the main design goals of many educational software tools [9].

Learning in games has been traditionally evaluated indirectly and separately from the actual gameplay; however, recently, researchers have realised that games can also serve as effective assessment tool [1], [11]. While players interact with a game, their behaviour is monitored and information about players' actions is collected, as well as feedback is 
provided in various forms. In general, this is similar to what educational assessment does, i.e., making inferences about learners' knowledge and skills by observing their actions in a specific context [1].

Various methods have been proposed to perform gamebased assessment (GBA) [12], [13]. The most commonly used methods are external (after gameplay) and internal (in-game) assessments [14], [15]. Internal assessment is also called "stealth assessment" because it is integrated in a game with the aim of supporting learning, maintaining flow, and reducing anxiety during the assessment [11]. The last aspect is very important in terms of reducing negative experience related to knowledge assessment since this process is more associated with negative emotions. This could be one of the reasons, why the game industry has recently started to use methods of affective computing, especially, when it comes to the development of educational games [16]. Therefore, the next section pays attention to the adaptation to emotions of learners in games focusing particularly on the assessment.

\section{GAME ADAPTATION TO USERS’ EMOTIONS}

It is considered that playing games is an emotional process rather than a rational one [17]. When playing an educational game, various emotional states can arise in the learning process. For example, positive emotions are experienced when tasks or challenges are completed. If knowledge assessment as a phenomenon is considered part of an educational game, then negative emotions (e.g., anxiety or even fear) can appear as well [6]. For example, anxiety can be felt if a learner is not confident about his/her knowledge level or a selected challenge level does not match appropriately learner's skills. Another negatively valanced emotional state - frustration usually occurs when a learner makes mistakes, gets stuck, or when important goals are blocked [18]. However, it should be noted that frustration is part of many games that leads to a challenging and enjoyable overall experience. Research shows that players are not spending long hours playing a game just to entertain themselves since most of the games can be very frustrating [19]. Therefore, it is considered that people play games to satisfy some basic needs, e.g., a need to learn, need to be challenged, or need to win.

As mentioned before, every learner has his/her own interests, needs, knowledge level, personality, etc., and all these characteristics can be analysed to provide adaptation in learning settings. Since the main goal of adaptive educational games is to support a broad range of learners, the achievement of this goal mainly depends on the system's ability to measure in relevant learners' characteristics an accurate way, including their emotional states, and to use these data to improve the learning process [8]. Results of knowledge assessment can also serve as a piece of data for the adaptation of various game elements, e.g., form of scaffolding and hints or appropriate challenge levels [1].

An easy and straightforward way of achieving adaptivity is to apply a direct mapping of threshold values of measured signals or extracted emotional features to values of game features or elements (e.g., challenge level, solving time, etc.)
[17]. By adapting different game elements, e.g., feedback, amount of help, and challenge level, it is possible to acquire higher player's satisfaction and keep him/her in the flow state, which is considered to be an optimal state for learning. Ideas about the flow state are based on the flow model developed by Csikszentmihalyi [20]. In the flow model, occurrence of some emotions such as boredom or anxiety shows mismatch between a challenge (a task difficulty level) and a knowledge level. Therefore, occurrence of such emotions can help identify, for example, knowledge gaps. If a player continues playing and his/her abilities improve but the challenge level stays the same, he/she will soon get bored. Therefore, to facilitate a flow state, a challenge and abilities must be carefully balanced to accomplish this type of adaptivity [1].

Emotions are closely related to motivation and, in general, two potential player's behaviours related to motivation can be distinguished - punishment avoidance and reward acquisition. In other words, motivation is either to achieve a reward or to try to reduce a punishment depending on goals, which a player sets for him/herself. These two behaviours can be explained by the achievement goal theory [21], in which $2 \times 2$ types of goals are defined: mastery goals and performance goals, each in terms of approach (or acquisition) or avoidance. As a result, the goals could be classified in the following way: performance-approach (e.g., it is important for the player to do better than others), performance-avoidance (e.g., it is important for the player to avoid performing worse than others), mastery-approach (e.g., the player is willing to learn as much as possible), and mastery-avoidance (e.g., the player does not want to perform worse than he/she did before). A game environment can be adjusted taking into account learner's goals, emotions, engagement level, motivation, and actions. Bontchev (2016) has identified three types of mechanisms to provide dynamic emotion-based adaptation in games [16]: 1) adjustment of explicit, implicit, or playerdriven game tasks and their managed appearance in the game flow (game content sequencing, provision and adaptation of automatic help, etc.); 2) adaptation of game difficulty to meet player's anxiety or a skill level based on performance; 3) adjustment of audio-visual game elements.

The analysis of the latest research shows that regardless of numerous studies that link together emotions and games [22], [23] only in rare cases emotions are analysed for the adaptation purposes. The mathematics game called Heroes of Math Island includes an affective agent (personified by a monkey character) that uses emotional expressions to respond to situations in the game [24].

Another game CRYSTAL ISLAND developed for microbiology education uses affective data to adjust a guidance level [25], [26]. Although there are educational games with emotion recognition and adaptation, none of them are developed with the aim of assessing directly learner's knowledge. Therefore, the authors of the present paper have made an additional analysis of existing environments supporting GBA to find out the current situation on capabilities of such environments to adjust behaviour to learner's emotions. 
Physics Playground is a 2D educational video game developed to measure knowledge in physics [15]. Experiments are carried out to observe learners' affective states and ontask/off-task behaviours during their interactions with the game and their influence on learning outcomes [8]. Although this game does not analyse affective states for adaptation purposes, the game developers are proposing possible responses that the game could perform when learners experience negative emotions. Therefore, these are only recommendations for potential adaptation.

Similar research is carried out in [27] with the game called Text Persuasion developed to assess learners' argumentation skills. The authors of the game have explored a relationship between GBA and cognitive/affective user experiences to examine which emotions are occurring during assessment and how these emotions are related to performance. Similarly to the previous example, emotions in this game are not used further to change game's course.

Another game called Semideus Exam measures math knowledge using the embedded assessment. During a gameplay, affective outcomes are also evaluated via measurement of learners' test anxiety and flow experience. Experiments with the game show that GBA lowered test anxiety and increased engagement leading to increased cognitive outcomes [11]. However, like in both previous examples, emotions are not processed with the aim of providing adaptation.

To sum up, educational games have a potential to adapt to individual learners by matching together game elements and learner's actions, performance, and learning progress, as well as emotions. Adaptation is a typical capability of intelligent tutoring systems which are created with the aim of providing personalized instruction for an individual learner to improve learning process and learning outcomes. Educational games also need personalization. Therefore, the next section introduces an adaptation approach developed by the authors for educational games supporting affective experiences.

\section{THE ADAPTATION APPROACH FOR AFFECTIVE GAMEPLAY}

In general, instructional adaptation can be provided at the macro- and micro-level. The former is implemented prior to learning on the basis of static player's data available to the system (or game) before learning (gameplay). The latter is ensured during the learning/teaching process based on dynamic player's data acquired in real time during the gameplay, e.g., based on responses, actions, emotions, etc.

\section{A. Macro-level Adaptation}

To provide the macro-level adaptation, learner's personality represented as Big Five personality traits (OCEAN) is analysed [28]. The personality traits give information not only about the learner's personality but also about his/her default mood or tendency to some specific emotions, preferred learning style, teacher's type, and teaching approach, as well as they allow identifying tendency towards goal achievements. The macro-level adaptation process is represented in Fig. 1.

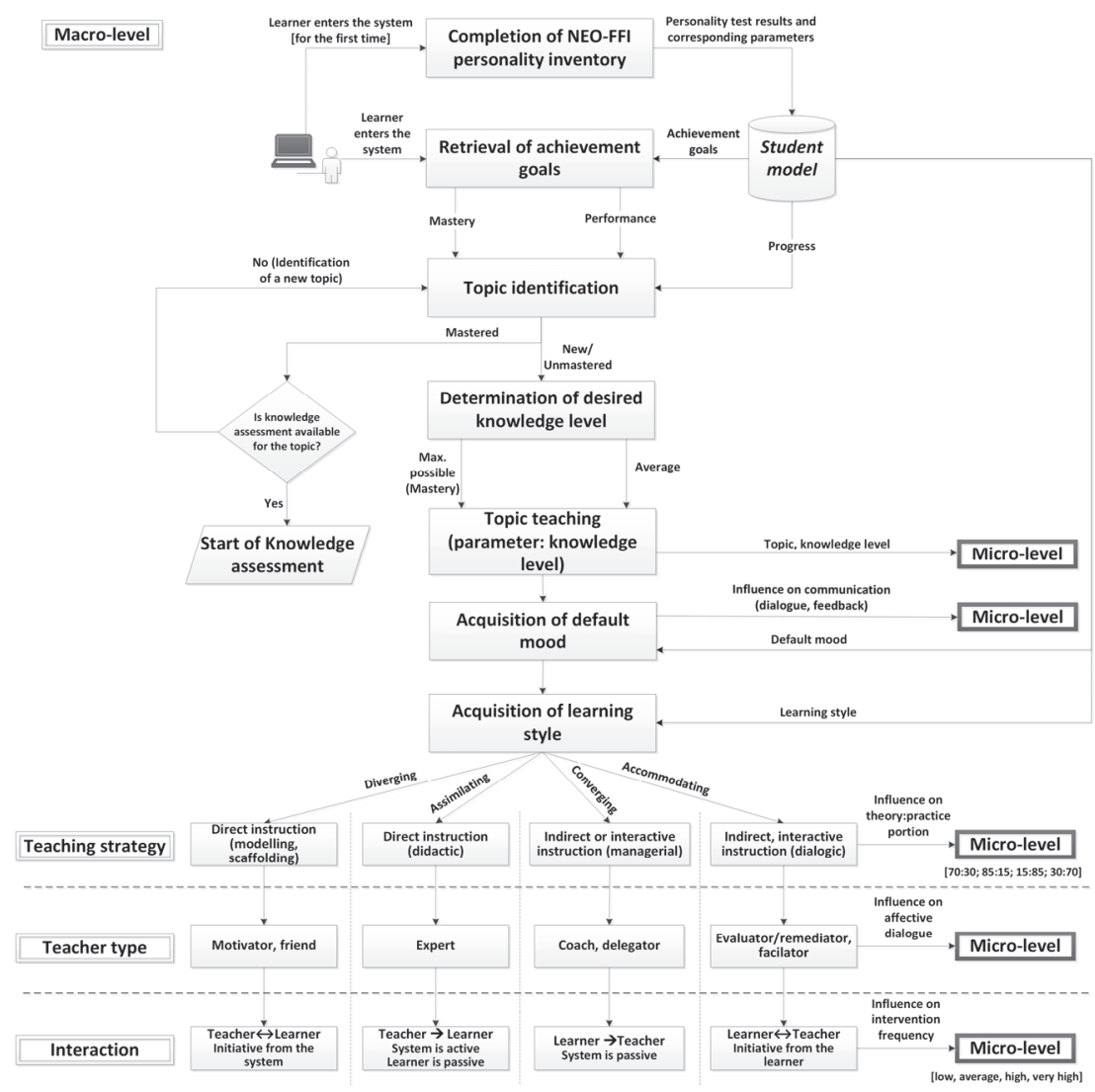

Fig. 1. Adaptation at the macro level. 
To ensure adaptation at the macro-level, the personality inventory is offered to the learner when he/she enters the system for the first time. The acquired personality traits are stored in the database (student model) together with accompanying parameters - identified learning style, teacher's type, teaching strategy, achievement goals, and default mood. The default mood is calculated on the basis of formulas provided by Mehrabian [29] to convert OCEAN values into Pleasure, Arousal and Dominance space. This information allows acquiring the personality's influence on emotions.

After storing all parameters, a topic is identified on the basis of progress and the learner's desired knowledge level is defined taking into account achievement goals. If a topic is mastered and there is an available knowledge assessment for it, then the learner can proceed with the assessment process. Otherwise, the teaching process is initiated to reach the defined knowledge level - either the maximum level for mastery oriented learners or the average level for performance oriented learners since they prefer to move faster through the learning process. Additionally, such parameters as a learning style, corresponding teaching strategy, and teacher's type are obtained. After the macro-level adaptation, the game initiates a micro-level adaptation, which is described next.

\section{B. Micro-level Adaptation}

As a gameplay starts, the micro-level adaptation is implemented on the basis of dynamic parameters. Emotions are considered one of the parameters, since they are occurring and changing during the gameplay. The next paragraphs explain the main steps of instructional process - topic teaching, practice, and knowledge assessment. The latter step is described in detail.

When topic teaching is initiated, first, the amount of theory is considered. If it is minimal, then a brief introduction is given, otherwise, theory is provided together with demonstrations (demo level). Demonstrations can be shown with or without learner's involvement. If the learner is also engaged in activities, then feedback on actions is provided depending on the teacher's type and action results. Emotion monitoring is ensured during the whole teaching process and, if negative emotions are detected, then based on their analysis and teaching situation, teaching is either repeated (e.g., if confusion is detected), modified (e.g., boredom), or carried on (e.g., if the teaching situation is frustrating but it is required for continuing learning). Positive emotions are promoted by giving positive or motivational feedback.

Depending on results of the teaching step, practice in the form of various challenges (game levels) comes next. If theory was minimal, more emphasis is put on practical training, during which some theoretical information can be provided in the form of tips. Furthermore, depending on a task and a difficulty level, additional options (such as help) are available. The learner can use these options to solve a task but it is important to remember that this will affect the result in terms of achievements/score. During the practice, learner's performance and actions are analysed (time required for problem solving, asking help, correctness of solution, etc.) and depending on the teacher's type different interventions are carried out by the system. In addition, emotions are evaluated

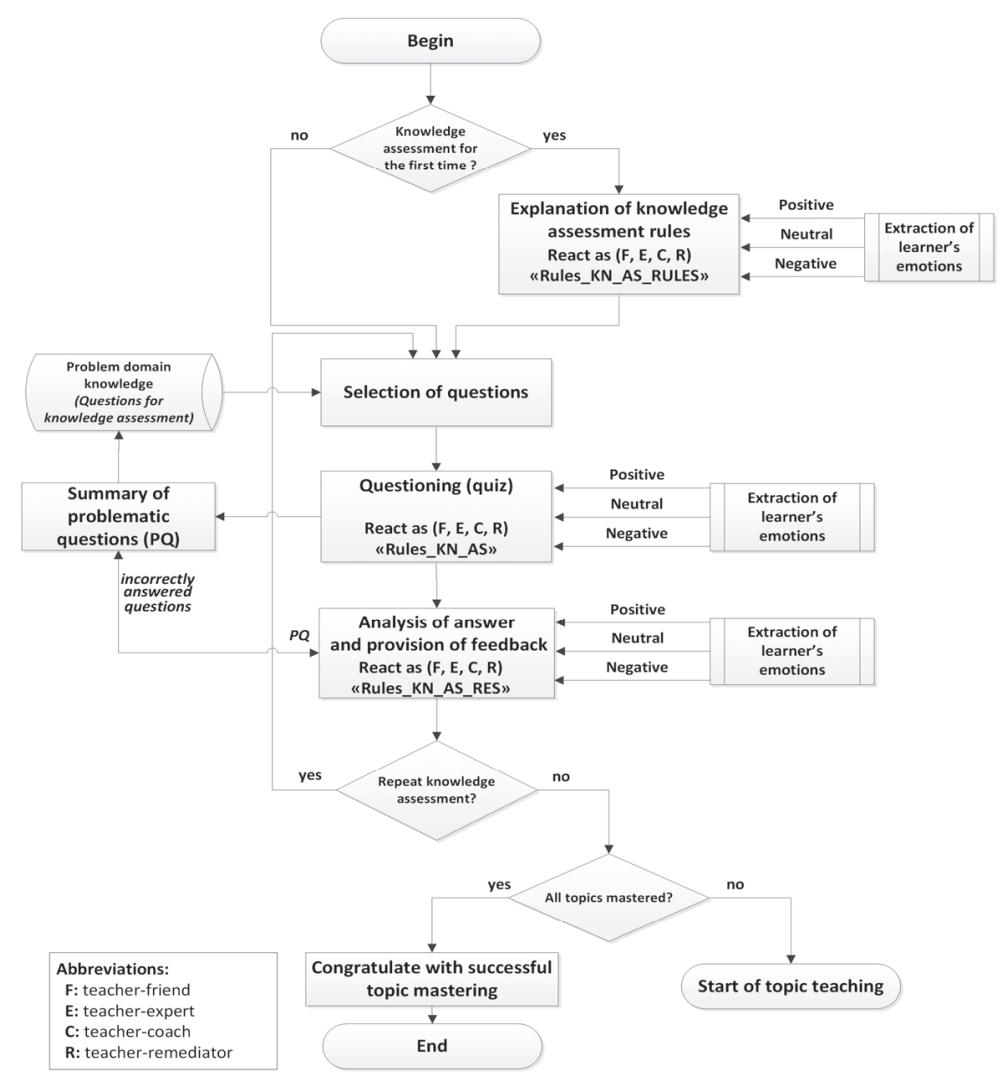

Fig. 2. Activities of knowledge assessment. 
to identify situations or system's actions that caused a negative experience and facilitated a positive one. Negative emotions can be a sign of missing knowledge, help required, or too low challenge level. Therefore, timely system's response is required to reduce their negative effect. After finishing the practical training (when the desired knowledge level is reached), the learner can start knowledge assessment. All the main activities of this step are demonstrated in Fig. 2.

In this case interventions are not carried out during the knowledge assessment itself but afterwards since knowledge is typically evaluated during exams when learners should only use their own knowledge without the help of others. An exception is a case if negative emotions are detected in a longer period of time or time needed for the provision of a correct answer took longer than an average time needed to answer a question. Otherwise, interventions could promote emergence of negative emotions (e.g., anxiety or frustration). After finishing assessment, results are analysed and feedback is given both on incorrect answers and on questions in which negative emotions were detected. Overall, all activities stay the same in this step and they are not changed according to the learning style or assigned teacher's type. The main differences appear in the adaptation of system's activities after the assessment and minimal changes - during the process itself (e.g., if a learner is not concentrating on the assessment, the system is trying to regain learner's attention).

Returning to Fig. 2, knowledge assessment starts with the activity that determines whether the assessment is carried out for the first time or not. If it is done for the first time, then the next activity is related to the explanation of knowledge assessment rules. Thus, the system intervenes in this step to explain rules. Learner's emotions are also monitored to adjust the explanation process or to make some changes in the system's behaviour. Adaptation or reacting rules for one of the teacher's types - "teacher-friend" - are provided further in the paper following with the more general explanations for others.

TABLE I

REACTING RULES FOR “TEACHER-FRIEND” IN KNOWLEDGE ASSESSMENT

\begin{tabular}{|c|c|}
\hline Identifier of Rules & Reacting Rules \\
\hline Rules_KN_AS_RULES (Friend) & $\begin{array}{l}\text { IF 'happy' OR 'interested' OR 'surprised' OR 'neural' THEN 'continue explanations' } \\
\text { IF 'sad' OR 'bored' THEN 'encouraging response' AND 'information about available options as help' } \\
\text { IF 'confused' THEN 'repeat explanations' } \\
\text { IF 'anxious' THEN 'information about available options as help' AND 'continue explanations' } \\
\text { IF 'frustrated' THEN 'calming down response' AND 'information about available options as help' }\end{array}$ \\
\hline$S$ (Friend) & 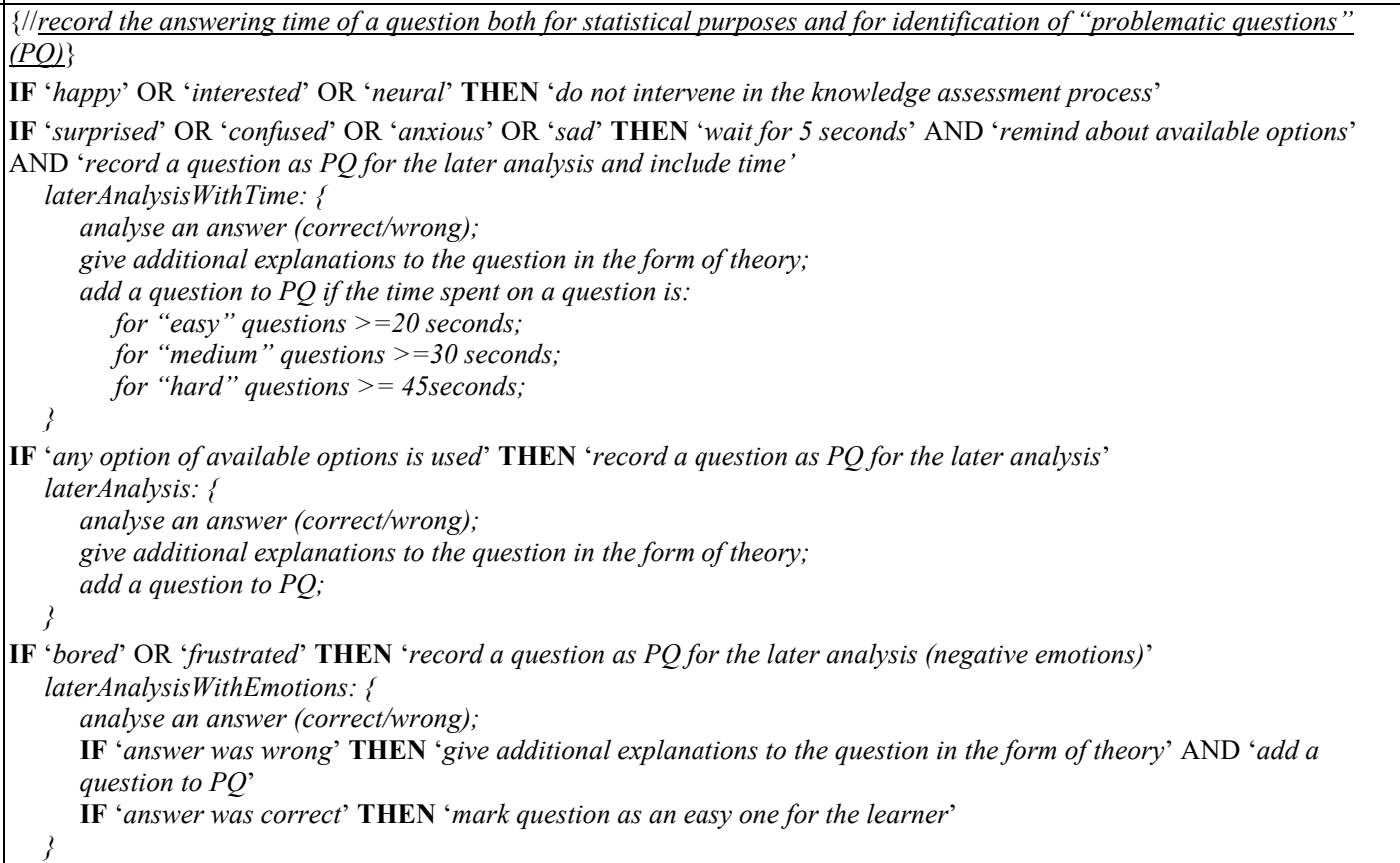 \\
\hline Rules_KN_AS_RES (Friend) & $\begin{array}{l}\text { IF 'answer was correct' AND 'questions }==P Q \text { ' THEN 'give explanations about a question' } \\
\text { IF 'answer was correct' AND 'happy' OR 'interested' OR 'surprised' OR 'neural' OR 'confused' OR 'anxious' OR 'sad' } \\
\text { OR 'bored' OR 'frustrated' THEN 'positive congratulations' } \\
\text { IF 'answer was wrong' AND 'happy' OR 'interested' OR 'surprised' OR 'neural' OR 'confused' THEN 'give explanations } \\
\text { about a question' AND 'add a question to PQ' } \\
\text { IF 'answer was wrong' AND 'anxious' OR 'sad' OR 'bored' OR 'frustrated' THEN 'motivating, encouraging response' } \\
\text { AND 'give explanations about a question' AND 'add a question to PQ' } \\
\text { IF 'result }<\text { minimum required' THEN 'repeat knowledge assessment' } \\
\text { IF 'result } \geq \text { minimum required' AND 'result < maximum possible' THEN 'offer to repeat knowledge assessment' }\end{array}$ \\
\hline
\end{tabular}




\section{Knowledge assessment "Teacher-friend"}

Reacting rules for this teacher's type are represented in Table I. For the previously mentioned activity (explanation of rules), the system should follow rules labelled as "Rules_KN_AS_RULES (Friend)" to provide adaptation to learner's emotions. The next activity is a selection of questions to assess learner's knowledge. After this activity, the selected questions are offered to a learner and the system follows rules named "Rules_KN_AS (Friend)".

In this step, learner's emotions and time spent on answering questions are an important source of information to identify "problematic questions" (PQs) that need further analysis. For example, if the system identifies that the learner feels anxious with a specific question and the emotional state does not change in 5 seconds, then the system automatically adds a question to PQs to analyse the answer to it in the next step. Additionally, questions which required using available options for answering are also recorded as PQ. After submitting, the answer is analysed and depending on its correctness and learner's current emotions feedback is given to the learner by following rules defined as "Rules_KN_AS_RES (Friend)". If a learner does not obtain the minimum required score for passing an assessment, then the system will automatically repeat the assessment process. If the assessment is successful but it is possible to get a higher score, then the learner will be offered to repeat the assessment process and his/her choice will be recorded to improve the system's performance in the future. If the learner refuses to repeat the assessment, then the system checks if the learner has mastered all topics available in the system. If there are still unknown/uncompleted topics, then the system proceeds with topic teaching. Otherwise, the system congratulates the learner with successfully completed learning.

\section{Knowledge assessment "Teacher-expert"}

For this teacher's type, an emphasis is not placed so much on emotions in comparison with "teacher-friend". The system is fully responsible for all decisions regarding knowledge assessment (to repeat it or not). Therefore, the main difference here lies in the activity related to the analysis of answers and feedback provision. Moreover, for learners with this teacher's type it is very important to understand why they made mistakes and they should receive detailed explanations on them. Furthermore, the system will repeat knowledge assessment automatically if the achieved result is not close to the maximum score since mastery is a goal for such learners.

\section{Knowledge assessment "Teacher-coach"}

In this case, the system gives control over the assessment process to the learner. Therefore, the system just once explains assessment rules and does not analyse learner's emotions (e.g., confusion) for repetitions. As the learner is fully responsible for his/her actions, if needed, he/she can request repetition of rules at any moment. This activity is followed by the selection of a question and its offering to a learner similarly as in the previous cases. The main differences appear in the activity related to the analysis of answers and feedback. Like the previous teacher's type, learners with this teacher also need detailed explanations on the mistakes made. However, if the learner has reached the minimum required score but not the highest possible one, then the system just motivates to re-take the assessment to get a higher score and the learner can decide on his/her own. In addition, the system records learner's decisions to improve its performance in the future.

\section{Knowledge assessment "Teacher-evaluator/remediator"}

For this teacher's type, the system mainly gives control over knowledge assessment to the learner but it still monitors his/her emotions. In this case, learner's emotions are analysed to adjust system's behaviour. If positive emotions are detected, no interventions are made during the knowledge assessment process. If negative emotions are identified, first, the system waits for changes in the emotional state. If emotions remain negative, the system reminds the learner about possible options (if they are available) and records a question as PQ. If the learner has used any of the options, the question automatically is added to PQ. After submitting the answer, the system makes its analysis. Depending on the correctness of the answer, question status (PQ or not), and learner's emotions, feedback is provided to a learner. As in the previous case, the learner is being motivated to repeat knowledge assessment if a possibility exists to get a higher score. Learner's decisions to repeat (or not) are being recorded with the aim of anticipating learner's choices in the future.

\section{IMPLEMENTATION OF THE ADAPTATION APPROACH}

Currently, the development of a 2D game is carried out as part of an affective tutoring system created for the study course "Fundamentals of Artificial Intelligence". The game is designed to provide double functionality - learning/practice through topic teaching and game levels (see Fig. 3) and knowledge assessment (see Fig. 4).

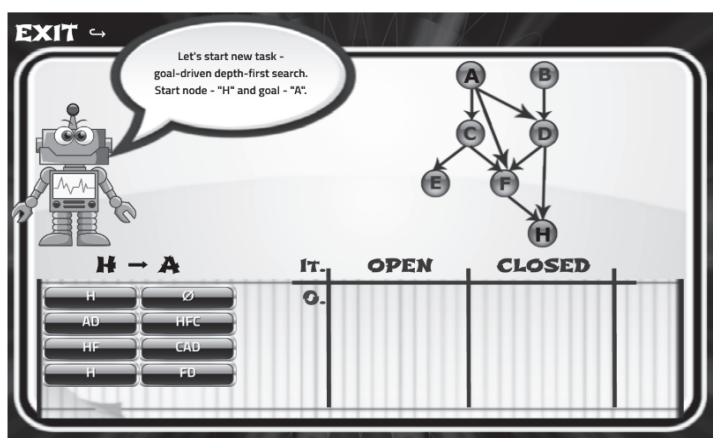

Fig. 3. Game interface and learning/practice activities.

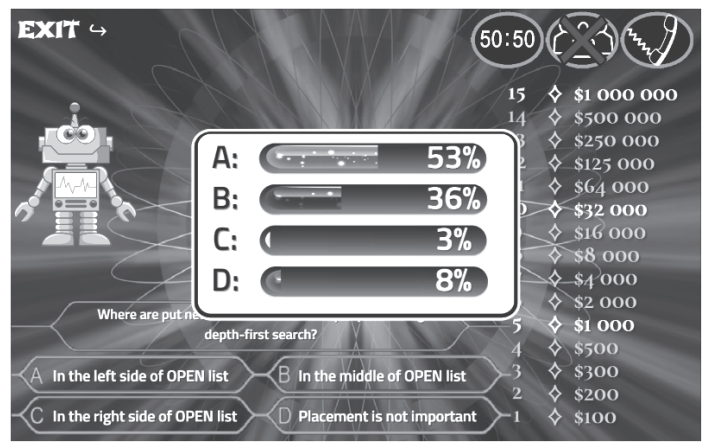

Fig. 4. Game interface for knowledge assessment. 
To provide adaptation at the macro-level, the system calculates personality traits based on learner's answers and feeds these data to the two neural network models, which assign a learning style and an achievement goal for the learner. For example, if personality traits have already been calculated (see Fig. 5) then using models the corresponding learning style and achievement goal can be identified. Both models were acquired on the basis of the collected surveys on learners' personalities, learning styles, and achievement goals.

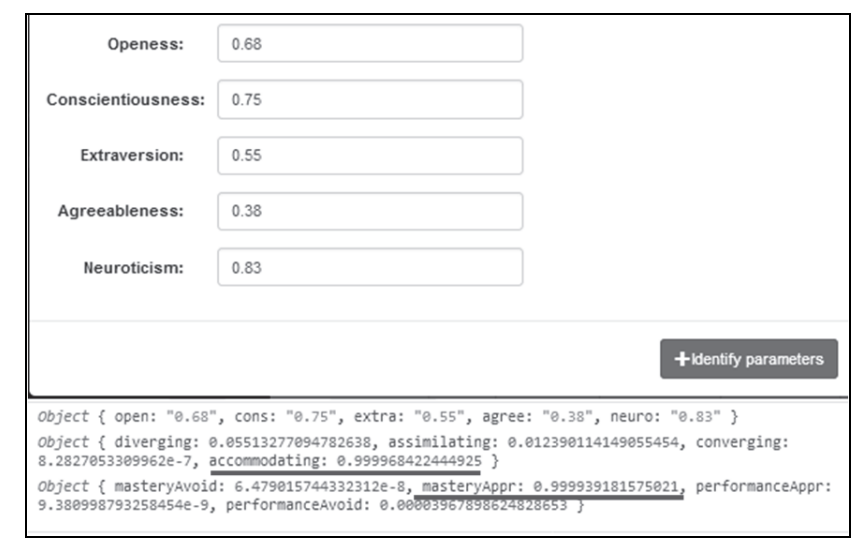

Fig. 5. Identification of learning style and achievement goal.

Emotion detection is implemented by adopting the existing web-based solution Emotion API developed by Affectiva [30]. This tool allows recognising various emotional states (e.g., joy, sadness, surprise and anger) based on the analysis of facial expressions acquired from camera stream. In addition, Emotion API gives information about activated facial actions, attention level and engagement in real time. Since not all learning specific emotions (e.g., frustration, confusion, boredom and anxiety) are recognised using this tool, studies regarding emotion identification were analysed to identify typical facial actions for these emotions. Neural network model is used for the classification of detected facial actions.

Knowledge assessment is implemented as a quiz following one of the most popular intellectual games called "Who Wants to Be a Millionaire?" in which players try to win \$ 1000000 by answering a series of multiple-choice questions of increasing difficulty. Although traditional assessments (e.g., multiple-choice questions) are considered non-motivating and not engaging for learners, as well as influencing negatively learning outcomes, recent research has suggested that modifications of these traditional assessments with more engaging features (e.g., involving gamification) is a promising approach to GBA [27].

\section{CONCLUSION}

Such concepts as game-based learning and assessment have been examined in the present paper. The analysis of the existing studies and developments has been carried out, first, to reveal the current situation in relation to the adaptation in educational games and, secondly, to identify how much learner's emotions are taken into account in educational games for adaptation purposes. The analysis made allows concluding that adaptation, which is considered to be an important aspect of the educational games to improve their effectiveness, is still implemented rarely and emotions as a source for further adaptation are used even rarer. However, educational environments designed for assessment should also analyse emotions because the assessment process is more associated with negative emotions, which could provide a negative impact on learning results.

Therefore, an adaptation approach has been introduced in the present paper, which allows considering characteristics of an individual learner at various adaptation levels (the macroand micro-level). Personality model is used at the macro-level providing data about a learning style and an achievement goal, as well as teacher's type and default mood are determined based on personality traits. This influences teacher's interventions (frequency, intervention type and content) at the micro-level and the achievement goal set for a learner during the assessment process. Furthermore, emotional states are identified with an aim of recognising potentially problematic questions and providing timely teacher's interventions in case of negatively valenced emotions or low attention level.

Currently, the first experiments with the system were conducted for its testing purposes and comparing assessment results of learners, who were using the game, with learners, who had paper-based version of the same test without involving the management of learner's emotions. Two more experiments with the system will be carried out during the next two months to gather more data and evaluate the effectiveness of the proposed approach in terms of learning outcomes (including exam results) and learners' emotional experiences.

\section{REFERENCES}

[1] V. Shute, F. Ke, and L. Wang, "Assessment and Adaptation in Games," Instructional Techniques to Facilitate Learning and Motivation of Serious Games, pp. 59-78, 2017. https://doi.org/10.1007/978-3-319-39298-1 4

[2] C. Conati and H. Maclaren, "Empirically Building and Evaluating a Probabilistic Model of User Affect," User Modeling and User-Adapted Interaction, vol. 19, no. 3, pp. 267-303, 2009.

https://doi.org/10.1007/s11257-009-9062-8

[3] H. van Oostendorp, E. D. van der Spek, and J. Linssen, "Adapting the Complexity Level of a Serious Game to the Proficiency of Players," EAI Endorsed Transactions on Serious Games, vol. 1, no. 2, pp. 1-8, 2014. https://doi.org/10.4108/sg.1.2.e5

[4] GlassLab, Inc., "SimCityEDU: Pollution Challenge!," 2014 [Online]. Available: https://s3-us-west-1.amazonaws.com/playfully-games/SC/ brochures/SIMCITYbrochure v3small.pdf

[5] N. Whitton, "Games as Reward Mechanisms," Digital Games and Learning: Research and Theory, pp. 99-108, 2014.

[6] A. Mavridis and T. Tsiatsos, "Game-Based Assessment: Investigating the Impact on Test Anxiety and Exam Performance," Journal of Computer Assisted Learning, vol. 33, no. 2, pp. 137-150, 2017. https://doi.org/10.1111/jcal.12170

[7] M. W. Eysenck and M. G. Calvo, "Anxiety and Performance: The Processing Efficiency Theory," Cognition \& Emotion, vol. 6, no. 6, pp. 409-434, 1992. https://doi.org/10.1080/02699939208409696

[8] V. J. Shute, S. D'Mello, R. Baker, K. Cho, N. Bosch, J. Ocumpaugh, M. Ventura, and V. Almeda, "Modeling How Incoming Knowledge, Persistence, Affective States, and In-Game Progress Influence Student Learning from an Educational Game," Computers \& Education, vol. 86, pp. 224-235, 2015. https://doi.org/10.1016/j.compedu.2015.08.001

[9] M. Prensky, "Fun, play and games: what makes games engaging?," in Digital Game-Based Learning, pp. 106-144, 2001. 
[10] E. Novak and T. E. Johnson, "Assessment of Student's Emotions in Game-Based Learning," Assessment in Game Based Learning: Foundations, Innovations, and Perspectives, pp. 379-399, 2012. https://doi.org/10.1007/978-1-4614-3546-4 19

[11] K. Kiili and H. Ketamo, "Evaluating Cognitive and Affective Outcomes of a Digital Game-Based Math Test," IEEE Transactions on Learning Technologies, 2017. https://doi.org/10.1109/tlt.2017.2687458

[12] J. Asbell-Clarke, E. Rowe, and E. Sylvan, "Assessment Design for Emergent Game-Based Learning," CHI '13 Extended Abstracts on Human Factors in Computing Systems on - CHI EA '13, pp. 679-684, 2013. https://doi.org/10.1145/2468356.2468476

[13] D. Eseryel, D. Ifenthaler, and X. Ge, "Alternative Assessment Strategies for Complex Problem Solving in Game-Based Learning Environments," Multiple Perspectives on Problem Solving and Learning in the Digital Age, pp. 159-178, 2011. https://doi.org/10.1007/978-1-4419-7612-3 11

[14] D. Ifenthaler, D. Eseryel, and X. Ge, "Assessment for Game-Based Learning," Assessment in Game-Based Learning, pp. 1-8, 2012. https://doi.org/10.1007/978-1-4614-3546-4_1

[15] V. Shute and M. Ventura, Measuring and supporting learning in games: Stealth Assessment. Cambridge, MA: The MIT Press, 2013.

[16] P. Wilkinson, "Affective Educational Games: Utilizing Emotions in Game-Based Learning," Proceedings of the 2013 5th International Conference on Games and Virtual Worlds for Serious Applications (VSGAMES), pp. 1-8, 2013.

https://doi.org/10.1109/vs-games.2013.6624219

[17] B. Bontchev, "Adaptation in affective video games: a literature review," Cybernetics and Information Technologies, vol. 16, no. 3, pp. 3-34, 2016.

[18] J. P. Gee, Good video games and good learning: Collected essays on video games, learning and literacy. New York: Peter Lang, 2007.

[19] K. Becker, "Pedagogy in Commercial Video Games," Games and Simulations in Online Learning. Research and Development Frameworks, pp. 21-47, 2007.

[20] M. Csikszentmihalyi, Finding Flow: The Psychology of Engagement with Everyday Life. New York: Basic Books, 1997.

[21] A. J. Elliott and H. A. McGregor, "A $2 \times 2$ Achievement Goal Framework," Journal of Personality and Social Psychology, vol. 80, pp. 501-519, 2001. https://doi.org/10.1037//0022-3514.80.3.501

[22] R. Ghali, S. Ouellet, and C. Frasson, "LewiSpace: An Exploratory Study with a Machine Learning Model in an Educational Game," Journal of Education and Training Studies, vol. 4, no. 1, pp. 192-201, 2016. https://doi.org/10.11114/jets.v4i1.1153

[23] N. Bosch et al., "Detecting Student Emotions in Computer-Enabled Classrooms," Proceedings of the 25th International Joint Conference on Artificial Intelligence, pp. 4125-4129, 2016.

[24] C. Conati and M. Gutica, "Interaction with an Edu-Game: A Detailed Analysis of Student Emotions and Judges' Perceptions," International Journal of Artificial Intelligence in Education, vol. 26, no. 4, pp. 9751010, 2016. https://doi.org/10.1007/s40593-015-0081-9

[25] J. Sabourin and J. Lester, "Affect and Engagement in Game-Based Learning Environments," IEEE Transactions on Affective Computing, vol. 5, no. 1, pp. 45-56, 2014. https://doi.org/10.1109/t-affc.2013.27

[26] R. Sawyer, A. Smith, J. Rowe, R. Azevedo, and J. Lester, "Enhancing Student Models in Game-based Learning with Facial Expression Recognition," Proceedings of the 25th Conference on User Modeling, Adaptation and Personalization - UMAP '17, pp. 192-201, 2017. https://doi.org/10.1145/3079628.3079686

[27] B. Lehman, D. Hebert, T. Jackson, and L. Grace, "Affect and Experience: Case Studies in Games and Test-Taking," in Proceedings of the 2017 CHI Conference on Human Factors in Computing Systems. Extended Abstracts, pp. 917-924, 2017. https://doi.org/10.1145/3027063.3053341

[28] S. Petrovica and M. Pudane, "Emotion Modeling for Simulation of Affective Student-Tutor Interaction: Personality Matching," Education and Information Technologies, vol. 10, pp. 159-167, 2016

[29] A. Mehrabian, "Pleasure-Arousal-Dominance: A General Framework for Describing and Measuring Individual Differences in Temperament," Current Psychology, vol. 14, no. 4, pp. 261-292, 1996. https://doi.org/10.1007/bf02686918

[30] Affectiva, "Products - SDK\&API," Affectiva, 2017 [Online]. Available: https://www.affectiva.com/product/emotion-sdk/

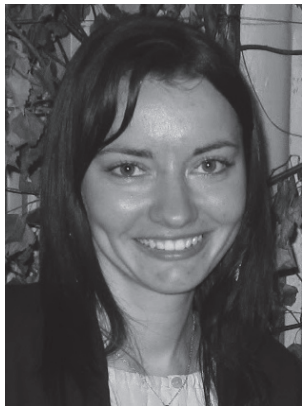

Sintija Petroviča received the Master's degree in Computer Systems from Riga Technical University, Latvia, in 2011.

She is a Doctoral student of the study program "Computer Systems" and works as a Research Assistant at the Department of Artificial Intelligence and System Engineering. She is developing her Doctoral Thesis related to the adaptation of tutoring process to student's emotions in the affective tutoring system. Her research interests include intelligent tutoring systems, game-based learning and affective computing.

E-mail: sintija.petrovica@rtu.lv

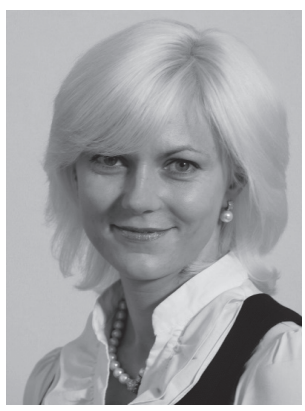

Alla Anohina-Naumeca is an Associate Professor at Riga Technical University with a fifteen-year experience of teaching in the field of computer science. She obtained the degree of Doctor in Engineering Sciences in the field of information technology in 2007 from Riga Technical University, Latvia. At the moment, she is developing her second Doctoral Thesis in the field of pedagogy. Her research interests include educational software, especially intelligent tutoring systems, and software solutions based on artificial intelligence. She has more than 60 publications and more than 20 research projects in the field of computer science, artificial intelligence, education, and educational software. She is constantly improving her knowledge in the fields of computer science and pedagogy by completing professional development courses and participating in workshops. Moreover, she is an active member of program committees of numerous scientific conferences.

E-mail: alla.anohina-naumeca@rtu.lv 